\title{
FDOT Reconstruction and Setting Optimization using Singular Value Analysis with Automatic Thresholding
}

\author{
Judit Chamorro-Servent ${ }^{1}$, Juan Aguirre ${ }^{1}$, Jorge Ripoll ${ }^{2}$, Juan J. Vaquero ${ }^{1}$ Member, IEEE , Manuel Desco ${ }^{1}$ Member, \\ IEEE
}

\begin{abstract}
Fluorescence Enhanced Diffuse Optical Tomography (FDOT) retrieves 3D distributions of fluorophore concentration in small animals, non-invasively and in vivo. The FDOT problem can be formulated as a system of equations, $d=W f$, where $W$ is a weight matrix that couples the measurements (d) to the unknown spatial distribution (f) of the fluorophore concentration (forward problem). The Singular Value Decomposition (SVD) of W has been previously employed to solve the inverse problem (image reconstruction) and to study the imaging performance of FDOT.

To achieve good image quality it is necessary to determine the number of useful singular values to retain. We use an automatic method that analytically calculates a threshold to select the significant singular values for SVD reconstruction of FDOT experiments previously validity in our laboratory.

Afterwards, this work appraises the effect of different settings of the acquisition parameters (distribution of mesh points, density of sources and detectors) of a parallel-plate noncontact FDOT, in order to achieve the best possible imaging performance, i.e., minimum number of singular values of $W$, maximum information content in acquired measurements and minimum computational cost.

We conclude that the use of a mesh with lower density in the direction perpendicular to the plates achieves better performance than the usual isotropic mesh points distribution. Any increase in the number of mesh points, sources and detectors at distances shorter than the photon mean free path leads to slight improvements in image quality while increasing computational burden.
\end{abstract}

Index Terms- Fluorescence, Diffuse, Optical, Tomography, regularization, U-curve, optimización, SVD, Singular, Value, Analysis

Manuscript received November 13, 2009.

This work is supported in part by Fundación Caja Navarra (\#12180), Ministerio de Ciencia e Innovación (TEC2008-06715 and TEC2007-64731) and EU-FP7 project FMTXCT-201792.

J. Chamorro-Servent, J. Aguirre, J.J. Vaquero, M Desco are with the Unidad de Medicina y Cirugía Experimental, Hospital General Universitario Gregorio Marañon, Madrid, Spain. (J. Chamorro-Servent email: jchamorro@hggm.es, M. Desco e-mail: desco@mce.hggm.es).

J. Ripoll is with FORTH, Institute of Electronic Structure Laser, Heraklion, Crete, Greece.

\section{INTRODUCTION}

Fluorescence Enhanced Diffuse Optical Tomography (FDOT) retrieves three dimensional distributions of fluorophore concentration in small animals, non-invasively and in vivo.

The FDOT problem can be formulated as a system of equations [1], $d=W f(1)$, where $\mathrm{W}$ is a weight matrix that couples the measurements (d) to the unknown spatial distribution (f) of the fluorophore concentration (forward problem). The Singular Value Decomposition method (SVD) of $\mathrm{W}$ [2] has been previously employed to solve the inverse problem (image reconstruction) and study the imaging performance on DOT and FDOT [3-6]. To achieve good image quality it is necessary to determine the number of useful singular values to retain. We use an automatic method, previously validated in our laboratory, that analytically calculates a threshold to select the significant singular values [7-8].

Afterwards, this work appraises the effect of different settings of the acquisition parameters (distribution of mesh points, density of sources and detectors) of a parallel-plate noncontact FDOT, in order to achieve the best possible imaging performance, i.e. using the minimum number of singular values of $\mathrm{W}$ to maximize the information content in acquired measurements while minimizing the computational cost.

\section{MATERIAL AND METHODS}

\section{A. Phantom}

We made a slab-shaped agar based phantom [9] using India ink and intralipid to obtain a reduced scattering coefficient of approximately $\mu \mathrm{s}=10 \mathrm{~cm}^{-1}$ and an absorption coefficient of $\mu \mathrm{a}=0.3 \mathrm{~cm}^{-1}$.

The size of the slab is $8.5 \times 6 \times 1.5 \mathrm{~cm}$. A capillary with its tip filled with $6 \mu \mathrm{l}$ of Alexa Fluor 700 (Invitrogen-Carlsbad, California) was inserted to the phantom, positioning the tip at the center of the slab.

\section{B. Noise threshold (regularization parameter)}

The U-curve [7-8] is a logarithmic plot of the sum of the reciprocals of the regularized solution norm, $\left\|_{\mathrm{f}}\right\|^{2}$, against the corresponding residual norm, $\left\|\mathrm{Wf}_{\lambda}-\mathrm{d}\right\|^{2}$ : 


$$
U \quad \operatorname{curve}(\lambda)=\frac{1}{\left\|\mathrm{Wf}_{\lambda}-\mathrm{d}\right\|^{2}}+\frac{1}{\left\|\mathrm{f}_{\lambda}\right\|^{2}}
$$

The optimum value of $\lambda, \lambda_{u}$, is given by the minimum of U-curve.

The procedure has been tested on experimental FDOT data collected with different experimental parameters for the slabpreviously described. These data were reconstructed by SVD [2], using Tikhonov regularization [10] with $\lambda$ parameters in the range $10^{-1}$ to $10^{-6}$. To confirm the sensitivity to the Ucurve cut-off value, we follow [8]: we verified that it falls into a heuristically acceptable range, experimentally defined as producing reconstructed images with a reasonable amount of noise, and simultaneously fulfills the Picard's condition [11]. Then, we verified that singular values $(\sigma)$, above the automatically calculated cut-off $\left(\lambda_{u}\right)$ decay to zero slower than the correspondent Fourier coefficients of $d\left(\left\|u^{r} d\right\|\right)$ when they are expressed in terms of the left singular vectors $\left(u_{i}\right)$ of W.

\section{Imaging performance optimization}

We constructed weight matrices of FDOT settings for the slab-shaped phantom described, with different density of sources, detectors, and distribution of mesh points. After decomposition into their singular values, we assessed:

(a) The influence on the imaging performance of the density of sources and detectors, using a $2 \times 2 \times 1.5 \mathrm{~cm}$ FOV, and 20x20x10 mesh points, and source and detectors FOV of $2 \times 2 \mathrm{~cm}$.

(b) The influence of the number of the mesh points and their spatial distribution, for a FOV of $1.5 \times 1.5 \times 1.5 \mathrm{~cm}, 12 \times 12$ detectors and 10x10 sources equally spaced.

\section{RESULTS}

Figures 2-5 shows our results on imaging performance based on the study of number of useful singular values (those above the calculated threshold).

\section{A. Influence on the imaging performance of the density of} sources and detectors

In Figures 2 and 3 we can see the influence of the density of sources and detectors on imaging performance:

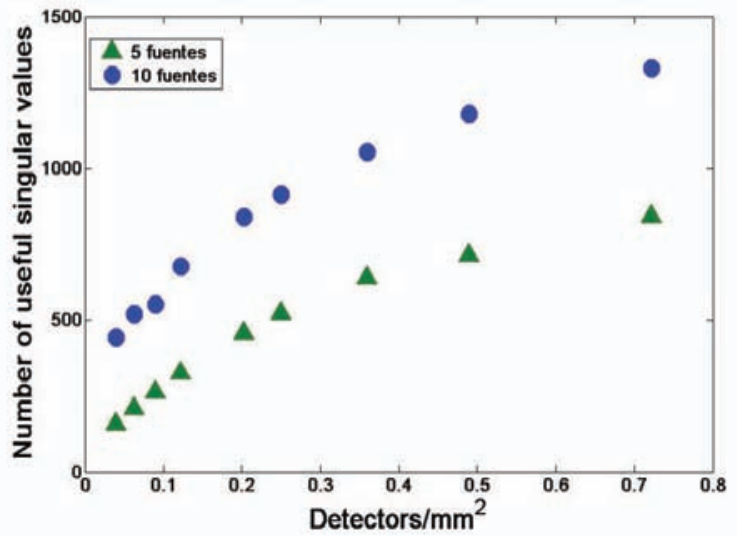

Fig. 2. Singular Value Analysis of the influence on the imaging performance of the density of detectors

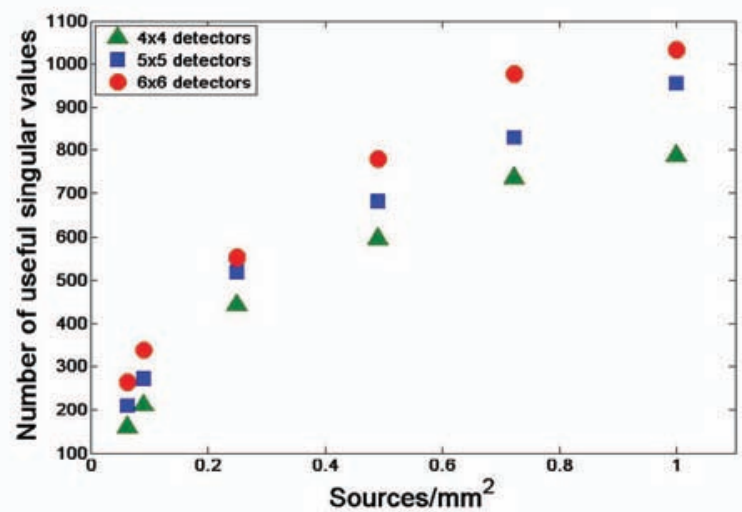

Fig. 3. Singular Value Analysis of the influence on the imaging performance of the density of sources

B. The influence on the imaging performance of the density of the number of the mesh points and their spatial distribution

In Figures 4 and 5 we can see the influence of the number of the mesh points and their spatial distribution on imaging performance:

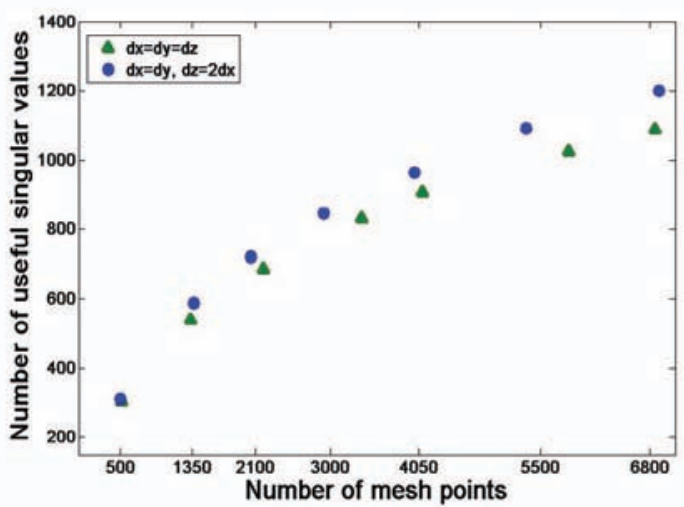

Fig. 4. Singular Value Analysis of the influence on the imaging performance of changing the isotropy of the voxel 


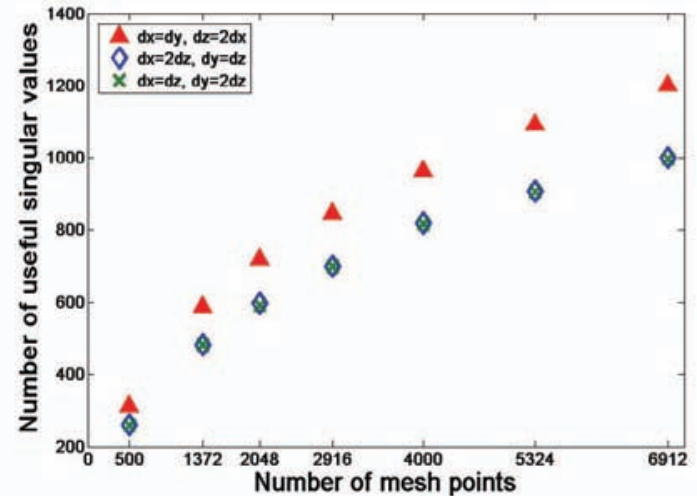

Fig. 5. Singular Value Analysis of the influence on the imaging performance of changing the different parallelepiped anisotropies of the voxels

\section{DISCUSSION}

The selection of useful singular values to retain was automatically assessed by the U-curve method in all the cases.

In the evaluation of the imaging performance, we can see (Fig.2) that for a fixed number of sources, the number of useful singular values, as a function of the detectors number quickly increase at the beginning but stabilizes when detectors are at distances closer than the photon mean free path (density of $1 \mathrm{~mm}^{2}$ ). Similar results appear (Fig.3) when fixing the detectors number and varying the number of sources.

Thus, any increase in the number sources and detectors at distances shorter than the photon mean free path leads to slight improvements in image quality while increasing computational burden.

Regarding the influence of the number of voxels and their spatial distribution, we can see (Fig.4) that a mesh density $\mathrm{dx}=\mathrm{dy}, \mathrm{dz}=2 \mathrm{dx}$ achieves better performance than an isotropic mesh density $d x=d y=d z$. In Fig. 5 we can also see that a mesh density $d x=d y, d z=2 d x$ is better than $d x=2 d z, d y=d z$ or $d x=d z$, $d y=2 d z$, where $z$ is the direction perpendicular to the plates.

These results achieve us to think that the use of a mesh with lower density in the direction perpendicular to the plates achieves better performance than the usual isotropic mesh point distribution used in most FDOT experiments.

\section{CONCLUSION}

These results seem to indicate that the use of a mesh with lower density in the direction perpendicular to the plates achieves better performance than the usual isotropic mesh point distribution.

The results obtained from the study of imaging performance can guide the selection of optimum acquisition parameters for any specific FDOT experiment.

\section{REFERENCES}

[1] O'Leary, M.A., Imaging with diffuse photon density waves. University of Pensylvania. Thesis doctoral, 1996.

[2] Golub, G.H. and C.F. Van Loan, Matrix computations. 1996: Johns Hopkins University Press. 694.

[3] Culver, J.P. and V. Ntziachristos, Optimization of optode arrangements for diffuse optical tomography: A Singular-value analysis. Optics Letter, 2001. 26(10): p. 701-703.

[4] Graves, E.E., et al., Singular-value analysis and optimization of experimental parameters in fluorescence molecular tomography. J Opt Soc Am A Opt Image Sci Vis, 2004. 21(2): p. 231-41.

[5] Graves, E.E., et al., A submillimeter resolution fluorescence molecular imaging system for small animal imaging. Medical Physics, 2003. 30(5): p. 901-912.

[6] Lasser, T. and V. Ntziachristos, Optimization of 360 degrees projection fluorescence molecular tomography. Med Image Anal, 2007. 11(4): p. 389-99.

[7] Krawczyk-Stando, Regularization parameter selection in discrete ill-posed problems-the use of the U-curve. Int. J. Appl. Math. Comput. Sci., 2007. 17(2): p. 157-164.

[8] J. Chamorro-Servent et al. An automatic method to select a noise threshold in the singular-value domain for reconstruction of parallel plate non-contact FDOT images. Abstract book of ESMI, 162, 2009.

[9] Cubeddu, R., et al., A solid tissue phantom for photon migration studies. Phys Med Biol, 1997. 42(10): p. 1971-9.

[10] Golub, G.H. and U.v. Matt, Tikhonov Regularization for Large Scale Problems. Workshop on Scientic Computing, 1997: p. 3-26.

[11] Hansen, The discrete Picard condition for discrete ill-posed problems. BIT, 1990. 30: p. 658-672.. 